\title{
New products and
}

\section{focus on restoratives and digital dentistry}

\section{BESPOKE ENDODONTIC SOLUTION}

The Optima MX INT system from BienAir provides a complete and reliable solution for your endodontic work.

Bien-Air's philosophy of 'click and work' is a new approach to your endodontic needs. The Optima MX is an interface that enables you to start treatment in a single click. This navigation system is impressively simple, allowing you to change the treatment settings in just a few clicks. You can perform your root treatments safely with a significantly reduced risk of instrument breakage due to the auto reverse function.
With an easy to use, intuitive display, the Optima MX INT converts your airdriven system to an upgraded electric operation; allowing, for example, NiTi endodontics to be covered with a standard 1:1 contra-angled handpiece. Thanks to the unique performance of the motor and control systems most other procedures are covered with just two contraangles (1:1 and 1:5), reducing the number of conventional instruments used in the surgery saving time, bother and expense. The system can be quickly and easily tailored to your individual requirements, offering a bespoke control system.

Reader response number 50

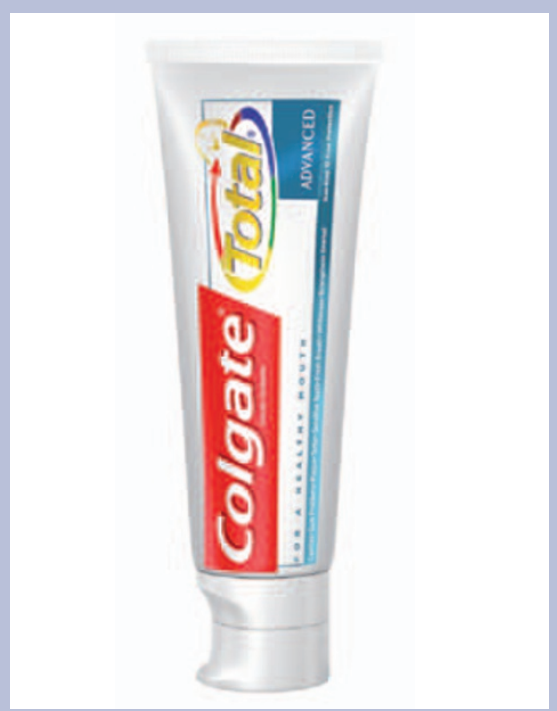

Now you can protect your patients from the most common and preventable dental problems by recommending new and improved Colgate Total toothpaste reducing plaque, calculus, dental caries, gum problems, sensitive teeth, enamel erosion, bad breath and staining.

Reader response number 51

\section{LIGHT SPEED RESTORATIONS}

Straumann have expanded their implant dentistry product portfolio to include digital solutions. Cadent iTero intra-oral scanning system and Straumann CARES CADCAM deliver real benefits to the dental team including cost savings, accuracy of impressions and final restorations in an exceptional choice of materials.

Cadent iTero intra-oral scanning system enables you to create an accurate 3D impression of the patient's teeth using a digital scanner, delivering considerable time and cost savings. Coupled with the Cadent iTero, Straumann CARES CADCAM produces a range of restorations including inlays, onlays, crowns and bridges in a wide choice of materials including titanium and zirconium.

By using these advanced systems, clinicians and technicians can work more efficiently and provide patients with accurate, well-fitting and aesthetic restorations.

Reader response number 52

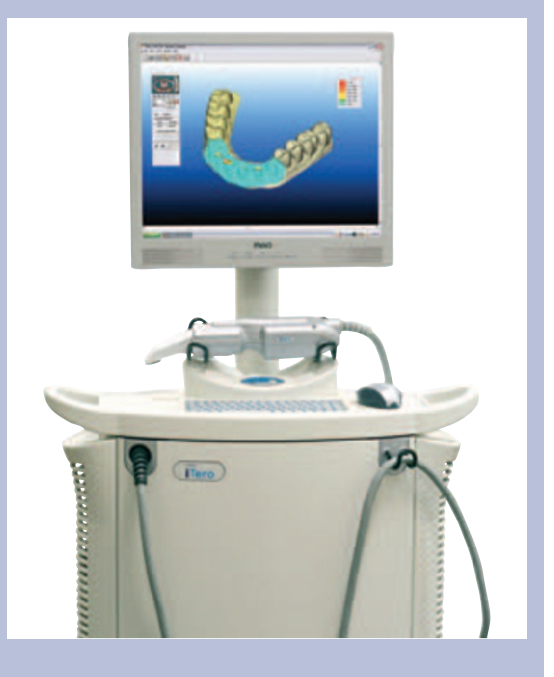

